\title{
A Percepção de Estudantes da Escola de Artes, Ciências e Humanidades da Universidade de São Paulo acerca da Disciplina de Resolução de Problemas I e II
}

\author{
Juliana Pedreschi Rodrigues*, Reinaldo Tadeu Boscolo Pacheco* \\ Escola de Artes, Ciências e Humanidades da Universidade de São Paulo \\ * Autores para correspondência: julianaprodrigues@usp.br, repacheco@usp.br
}

\section{RESUMO}

Esta pesquisa apresenta reflexões sobre a experiência de tutoria de estudantes de graduação no Ciclo Básico da Escola de Artes, Ciências e Humanidades da Universidade de São Paulo que cursaram as disciplinas de Resolução de Problemas I e Resolução de Problemas II, as quais se fundamentam na metodologia de aprendizagem baseada em problemas, o PBL (Problem-based Learning). Assim sendo, o objetivo principal do presente estudo foi demonstrar como se dá o referido processo de aprendizagem ativa e as peculiaridades desse tipo de estratégia ao longo de quinze semanas, além da comparação de tal metodologia com experiências de aprendizagens que os estudantes vivenciaram no Ensino Fundamental e no Ensino Médio. Nas considerações finais, discutem-se aspectos positivos e negativos observados nessa metodologia de aprendizagem ativa, as diferenças apreendidas pelos estudantes nos momentos de construção de seus conhecimentos e as particularidades da relação tutor/ estudante e estudante/estudante presentes ao longo do processo de aprendizagem por resolução de problemas.

Palavras-Chave: Metodologias Ativas; Aprendizagem Baseada em Problemas; Protagonismo Estudantil.

\begin{abstract}
This research presents reflections on undergraduate students tutoring experience in the Basic Cycle of EACHUSP (School of Arts, Sciences and Humanities of the University of São Paulo) who attended the discipline Problem-Solving I and II, established on learning methodology based on problems, PBL (problem-based learning). Therefore, the main objective of this study was to demonstrate how is this process of active learning and the peculiarities of this type of strategy over fifteen weeks beyond compare this methodology with learning experiences that students experienced in middle and high school. Through focus group aimed to understand the perceptions of students about his involvement with this methodological approach to learning. In concluding, remarks discuss the students' perception of this active learning methodology, the differences learned by students in times of building their knowledge and characteristics of tutor/ student and student/student relationship present throughout the learning process for resolution problems.
\end{abstract}

Keywords: Active Methodologies; Problem-based Learning; Student Leadership.

\section{Introdução}

A presente pesquisa analisou a percepção de estudantes da Escola de Artes, Ciências e Humanidades da Universidade de São Paulo que cursaram, no Ciclo Básico dessa instituição, a disciplina intitulada Resolução de Problemas I e II (RP I e RP II). Como objetivo principal, tentou-se identificar todo o processo de aprendizagem na referida disciplina, fazer a comparação de experiências educacionais anteriores, do Ensino Fundamental e do Ensino
Médio dos estudantes, relacionadas com o processo de construção de seus conhecimentos, e, a partir de tais experiências, sugerir possíveis mudanças.

Do ponto de vista do olhar docente, o que nos chama a atenção ao usar a Resolução de Problemas, que tem como base a metodologia de Aprendizagem Baseada em Problemas (ABP) ou PBL (Problem-based Learning), é o envolvimento e entusiasmo, por parte de vários estudantes, com as estratégias utilizadas. No entanto, em menor medida, mas também 
verificável na realidade didática, observa-se parte dos estudantes com dificuldades na compreensão da importância e do significado dessas estratégias para suas vidas acadêmicas (PARANHOS \& MENDES, 2010).

Diante de tal percepção docente, parte-se da hipótese de que a formação anterior desses jovens é um dos vetores de influência quanto a essa percepção estudantil no que se refere à importância das estratégias didáticas em Resolução de Problemas na sua formação. Assim sendo, os caminhos e resultados desta investigação, que serão destacados na conclusão do presente estudo, tiveram como ponto de partida o olhar atento ao processo de aprendizagem e às percepções dos estudantes sobre as estratégias de aprendizagem propostas pela disciplina Resolução de Problemas I e II.

Para a realização desta pesquisa, de natureza qualitativa, utilizamos a técnica do grupo focal (RESSEL et al., 2008), tendo como finalidade discutir e comentar a experiência pessoal dos estudantes selecionados, em questões referentes à aprendizagem, ao relacionamento interpessoal e à construção de conhecimento, e sobre as possíveis diferenças entre essa metodologia e a aprendizagem considerada "tradicional". Para tanto, reunimos um grupo de treze estudantes, de uma turma de cerca de sessenta, que cursaram a disciplina de RP I e RP II, com a finalidade de coletar informações que foram divididas em três focos. O primeiro foco buscou captar e comparar as diferenças na forma de aprendizagem ocorrida no Ensino Médio e suas experiências de aprendizagem em RP I e RP II na EACH/USP. $\mathrm{O}$ segundo foco pretendeu registrar a percepção de mudanças na relação estudante/tutor e estudante/ estudante, e o terceiro foco visou a compreender se foram, de fato, percebidas alterações significativas na forma de os estudantes aprenderem e produzirem novos conhecimentos. Ao final, considerando o protagonismo estudantil no processo, abrimos ao grupo a oportunidade de apresentação de sugestões para a melhoria dessas disciplinas.

Para o desenvolvimento desta investigação, apresenta-se um breve histórico da metodologia PBL e de sua utilização na EACH-USP.

\section{Sobre a Metodologia PBL (Problem-Based Learning)}

No Brasil, mesmo nos dias atuais, a metodologia Problem-Based Learning ainda é desconhecida e pouco utilizada nas universidades públicas, apesar da chegada das Tecnologias da Informação e Comunicação (TICs) e do debate recorrente sobre a necessidade de atualização das metodologias de ensino e aprendizagem. Como no restante do mundo, o PBL chega ao Brasil na área da saúde, em cursos de Medicina, e aos poucos ganha outros espaços.

Andrade (2005) afirma que essa metodologia promove a participação ativa, o desenvolvimento da capacidade crítica e a autoaprendizagem do estudante, com base na resolução de problemas. A autora também relata o surgimento desse modelo de aprendizagem nos cursos da área da saúde, na Escola de Medicina da Universidade de McMaster em Hamilton, Ontário, ao final dos anos de 1960 e início de 1970, e que, posteriormente, disseminou-se para outros países e diferentes cursos de graduação e pós-graduação.

Sobre essa metodologia, Ribeiro (2008) considera, fundamentada na Aprendizagem Baseada em Problemas (ABP), que tal método tem como recurso principal de aprendizagem a utilização de problemas do cotidiano como forma de "iniciar, motivar e focar a aprendizagem de conhecimentos conceituais, procedimentais e atitudinais".

A pesquisa realizada por Faustino (2013), que teve a área da saúde como lugar de observação, descreve o PBL como "uma estratégia educacional, centrada no aluno, que o ajuda a desenvolver o raciocínio e a comunicação, habilidades essenciais para o sucesso em sua vida profissional". Em sua pesquisa, que teve como objetivo levantar estudos disponíveis nas bases de dados virtuais Medline e Lilacs, publicados entre os anos de 2000 e 2010, sobre a utilização do PLB no Ensino Superior, a autora revela, dentre os principais resultados, que a avaliação positiva do PBL foi quase "unânime" entre os estudos analisados.

Pesquisas semelhantes, como as de Andrade (2005), Ribeiro (2008) e Miranda Junior (2016), também oriundas da área da saúde, defendem o 
PBL como uma metodologia inovadora no ensino de graduação e apresentam alguns aspectos fundamentais para o seu desenvolvimento. Por sua vez, Faustino (2013) considera a participação ativa dos estudantes no grupo tutorial como uma condição imprescindível durante todo o processo e que o problema, seja ele fictício ou real, deve ser continuamente "acompanhado por um tutor docente", que estimulará os estudantes durante as discussões a elaborarem hipóteses, objetivos e possíveis estratégias de solução.

Miranda Junior (2016) destaca ainda a necessidade de espaços específicos para as reuniões, com acesso à tecnologia como parte importante da aprendizagem, a importância de realizar a avaliação constante dos estudantes e a "promoção do trabalho em grupo e da cooperação de forma interdisciplinar e multiprofissional" visando a incentivar o protagonismo do estudante e a valorização do processo de aprendizagem, sempre pautado no desenvolvimento de "habilidades técnicas, cognitivas, de comunicação e atitudinais, promovendo assim o gerenciamento do aprendizado pelo próprio estudante".

Finalmente, ao abordar as limitações e dificuldades existentes nessa metodologia de aprendizagem, o autor ressalta as barreiras para a institucionalização do novo, do inovador, o desconforto de parte dos estudantes que encontram dificuldade para assumir uma postura proativa e responsável pelo seu próprio desenvolvimento e, também, o desconhecimento e inexperiência de grande parte dos tutores/ professores com a própria metodologia.

Vignochi et al. (2009), em artigo de revisão sobre o tema, refere que a metodologia PBL tem se mostrado um instrumento valioso na formação do profissional no campo da saúde, com vantagens sobre o método de ensino tradicional. Certamente, grande parte dessas estratégias podem ser aplicadas a qualquer campo da formação profissional.

No entanto, para a implantação de tal metodologia de aprendizagem, há necessidade de um considerável esforço institucional. São necessárias adaptações, tais como mudanças na forma de avaliação, mudanças na forma de ver o papel do docente no processo de ensino/aprendizagem, investimentos em infraestrutura, adaptações do ambiente, melhoria das bibliotecas, entre outros. Esse processo de mudança, portanto, traz o desafio de romper com modelos de ensino tradicional.

\section{O Uso do PBL na EACH-USP}

A Escola de Artes, Ciências e Humanidades da Universidade de São Paulo - EACH, popularmente conhecida como USP Leste, está localizada na Zona Leste da cidade de São Paulo e nasce em resposta às reivindicações de grupos populares organizados dessa região, que travaram, ao logo das décadas de 1990 e primeiros anos de 2000, um longo e difícil diálogo com os diversos setores da sociedade e do poder público em prol da transformação, para melhor, das condições de vida dos habitantes dessa região.

Diferentemente dos cursos mais antigos e considerados tradicionais na Universidade de São Paulo, os da EACH, em sua proposta pedagógica, decidiram por incluir em sua estrutura curricular, no primeiro da graduação, o Ciclo Básico (BRASIL, s.d.). Nos dois primeiros semestres de seus cursos são oferecidas disciplinas que possibilitam aos estudantes terem uma formação acadêmica baseada em propostas interdisciplinares e inovadoras, com o intuito de dar-lhes uma visão de fenômenos sociais de maneira crítica e a partir de diferentes perspectivas.

Em busca de uma aprendizagem diferenciada e inovadora, a estrutura curricular do Ciclo Básico da EACH foi organizada levando em consideração três eixos. Conforme Araújo e Arantes (2009), o primeiro eixo, Formação Introdutória Específica, propõe apresentar ao estudante as bases conceituais de seu curso e, desde o início, mostrar indícios e possibilidades da futura atuação profissional. Já o segundo eixo, denominado Formação Geral, visa a ofertar disciplinas gerais - multidisciplinares e interdisciplinares -, voltadas para uma formação abrangente, que contemple a "complexidade dos fenômenos naturais, sociais e culturais". Finalmente o terceiro eixo, de Formação Científica, por meio da disciplina Resolução de Problemas, tem como objetivo central incentivar a formação científica dos estudantes por meio da construção coletiva de novos conhecimentos, da 
integração de estudantes dos diferentes cursos e da aproximação com a comunidade local ${ }^{1}$.

No ciclo Básico as disciplinas de Resolução de Problemas I e Resolução de Problemas II, ambas baseadas nos princípios da metodologia $\mathrm{PBL}$, são ofertadas nos dois primeiros semestres dos cursos de graduação da EACH. Dentre outros objetivos, busca-se estimular no estudante o interesse pela pesquisa, a autonomia nos estudos, a criticidade e, especialmente, a capacidade de empatia e de desenvolver pesquisas em grupo. De maneira geral, para a organização e o desenvolvimento da disciplina de RP I, no Ciclo Básico, as turmas mistas são compostas por sessenta estudantes de diferentes cursos que, posteriormente, são divididos em quatro grupos de trabalho, com uma média de quinze estudantes cada, que finalmente são subdivididos em dois subgrupos, um de sete e outro com oito estudantes. No caso da disciplina de RP II, o procedimento é o mesmo, mas os cerca de sessenta estudantes do grupo-classe são oriundos do mesmo curso, o que permite aproximações com problemas específicos do campo de atuação profissional ${ }^{2}$. O cenário ideal e desejável é que os quatro docentes se dividam entre os oito subgrupos de trabalho, sendo dois grupos para cada docente/tutor. Nesse processo, é fundamental que todos os quatro docentes tenham a responsabilidade de fazer a apresentação das estratégias de trabalho da referida disciplina e do conteúdo do PBL, participem das discussões para definição do tema central ou gerador, dos subtemas e do processo de divisão dos subgrupos, de forma que todos tenham membros de diferentes cursos, no caso de RP I.

Ao longo de um semestre, além de ser responsável pela tutoria de seus dois subgrupos, o tutor/professor deverá deixar clara a estratégia de trabalho dessa disciplina que busca incentivar "o papel ativo do estudante na construção do conhecimento, por meio do trabalho em pequenos grupos e do desenvolvimento de pesquisas sobre problemas complexos relacionados às questões da atualidade ou a realidade de seu cotidiano" (UVINHA, 2009).

\section{O Processo de Aprendizagem no Ciclo Básico da EACH/USP}

No Ciclo Básico da EACH/USP, durante o processo de aprendizagem, diferentemente do modelo de ensino considerado "tradicional" desenvolvido, em geral, pela educação básica do país, de Ensino Fundamental e Médio, a prioridade nessa disciplina é favorecer o protagonismo do estudante em detrimento da presença, muitas vezes, diretiva e autoritária do professor.

Em uma reunião antes do início de cada semestre, geralmente, a coordenação de curso do Ciclo Básico sugere temáticas aos tutores, visando a direcionar o trabalho com os subgrupos. Esses temas podem surgir de problemas observados na comunidade, podem envolver questões sociais atuais e polêmicas, ou mesmo surgir da curiosidade e do interesse dos estudantes. Nos últimos anos, os grupos de tutores tiveram toda a liberdade de definir os mais diversos temas dentro de um mesmo grupo-classe, sem precisarem ficar presos a um "tema gerador".

A proposta do Ciclo Básico aponta como fundamental para o encaminhamento dos quinze encontros semanais de tutoria em RP I e RP II que, nos primeiros encontros com os grupos, os docentes construam com os estudantes os tópicos temáticos a serem estudados no semestre, por meio de uma ou duas aulas expositivas, com a intenção de dar aos estudantes uma visão mais profundada e compartilhada dos tópicos temáticos. Após essa etapa, parte-se para a definição de um problema, de hipóteses e das estratégias de estudos, pesquisas e intervenções que serão necessárias para responder ao problema, já nos grupos menores. Os grupos já subdivididos em dois subgrupos de sete e oito estudantes terão que cumprir, em forma de rodízio, durante as quinze semanas, tarefas diferentes envolvendo a coordenação das atividades de pesquisa, a distribuição de tarefas do grupo e o agendamento dos encontros. Para isso, junto ao professor-tutor, os grupos estabelecem as mais diversas estratégias de encontros e trocas de informação, a forma de avaliação de cada um dos encontros, utilizando-se até mesmo a formação de grupos virtuais nas redes sociais presentes na rede mundial de computadores. 
Para a organização do calendário semestral devem-se prever dois encontros para socialização do projeto de pesquisa, por volta da quinta semana, para apresentação e avaliação do pré-projeto, e um novo encontro, na décima quinta semana, momento em que os estudantes apresentarão os resultados da pesquisa e entregarão um relatório científico impresso registrando os avanços do grupo, que será avaliado por todos os tutores, momento em que estes também avaliam todo o processo de aprendizagem, autoavaliam-se e avaliam os seus pares.

Nos últimos anos, esse relatório científico tem sido realizado no formato de artigo científico, baseando-se sua escrita inclusive em normas das próprias revistas científicas selecionadas e com artigos lidos para cada tipo de problema. Deve-se destacar que, nos últimos anos, alguns desses trabalhos com inegável qualidade foram apresentados em eventos científicos fora da universidade e, portanto, avaliados por diferentes pares. Além disso, alguns também foram apresentados em eventos científicos da universidade, como a Semana da Ciência e o SIICUSP.

Faz-se necessário salientar que, apesar de a orientação do manual, elaborado pela comissão de coordenação do Ciclo Básico, prever, em geral, o encaminhamento das etapas de desenvolvimento da pesquisa em quinze semanas, o ritmo de trabalho e as estratégias dependem de cada tutor, que desenvolve à sua maneira esse processo, por terem maior ou menor aderência com o modelo de aprendizagem que essa metodologia propõe e mesmo conhecimentos sobre os princípios do Ciclo Básico.

\section{Considerações Finais}

Ao final das quinze semanas de observação contínua da aprendizagem baseada na resolução de problemas, fez-se uma reunião com um grupo com treze estudantes convidados, integrantes do grupo-classe de sessenta pessoas e que participaram de todo o processo de RP I e RP II. O critério para participação no grupo focal foi convidar um ou dois estudantes de cada um dos oito grupos, dentre eles aqueles que se sentiram bastante confortáveis com a participação em RP I e II e outros que se sentiram desconfortáveis. Após a utilização da técnica do grupo focal, as informações coletadas entre os estudantes convidados foram divididas em três focos que revelaram aspectos interessantes sobre as principais diferenças na forma de aprendizagem ocorrida no Ensino Médio e suas experiências de aprendizagem em RP I e RP II: sobre a percepção de mudanças na relação estudante/ tutor e estudante/estudante; sobre mudanças percebidas no modo de aprendizagem e produção de novos conhecimentos; sobre o protagonismo estudantil no processo. Além disso, houve oportunidade para que os estudantes deixassem sugestões para a melhoria no processo de aprendizagem. A partir dos dados coletados durante a técnica de grupo-focal, da leitura e da interpretação das falas dos estudantes, podem-se ressaltar os seguintes pontos para análise, conforma se observa na Tabela 1:

\section{Ensino Médio}

Conhecimento pronto

Não ensina a pesquisar

Conhecimento individualizado

Preparação para o vestibular

Ensino tradicional

Preponderância do conteúdo

Poucas cobranças e reprodução do conteúdo

\section{Resolução de Problemas I}

Buscar conhecimento

Preparação para novas disciplinas

Construção coletiva do conhecimento

Maior autonomia

Papel ativo do estudante

Trabalho coletivo

Pressão pelo resultado

Tabela 1 - Sobre a Aprendizagem no Ensino Médio e Suas Diferenças com a Estratégia da disiplina RP I. 
Observa-se que há um destaque na fala dos sujeitos com relação ao papel ativo na construção do conhecimento. Ficou bastante evidente a percepção dos alunos quanto às diferenças no processo de aprendizagem que viveram no Ensino Médio. Indagados sobre a possibilidade da estratégia de RP no Ensino Médio, alguns estudantes declaram que seria praticamente impossível, dada a estrutura dos grupos e a existência de salas numerosas nas escolas em que estudaram. A mudança na postura do educador escolar também é vista como um vetor de dificuldade, na medida em que os estudantes identificam essa postura no Ensino Médio como "autoritária e diretiva".

Na relação estudante/estudante evidenciou-se a dificuldade no trabalho coletivo e na divisão de tarefas. No caso da relação estudante/tutor, as falas dos entrevistados parecem indicar uma necessidade latente dos estudantes por um maior direcionamento e, contraditoriamente, alguns estudantes declaram que, embora a relação seja mais horizontalizada, alguns tutores acabam por impor determinados caminhos de construção do conhecimento. Observa-se que alguns estudantes parecem, de certa maneira, esperar por tal postura mais diretiva, como um reflexo do que viveram no Ensino Médio (c.f. Tabela 2).

Essas diferenças percebidas pelos estudantes na postura dos tutores podem ser decorrentes de diversos fatores, dentre eles: as diferentes experiências dos tutores com a metodologia; as diferenças de domínio de aspectos metodológicos no campo das ciências humanas; a aderência dos tutores ao tema geral. Tais diferenças de encaminhamento das etapas de desenvolvimento da pesquisa podem acabar por desmotivar os estudantes, já que, embora isso não seja estimulado, há dentro do grupo-classe atitudes comparativas com relação aos diferentes encaminhamentos pelos diferentes tutores.

Obviamente que não se trata de defender aqui uma postura "homogênea" dos tutores, mas se percebe que, mesmo para a valorização da singularidade pedagógica da relação com cada grupo, seria fundamental uma linguagem comum entre os tutores em relação à valorização do processo pedagógico e das etapas de encaminhamento da Resolução de Problemas, dando relevância a esse tipo de estratégia de construção do conhecimento.

Observa-se que os estudantes apresentam uma percepção mais positiva do que negativa do processo (c.f. Tabela 3). No entanto, apesar de entenderem que o processo se dá de forma bastante desafiadora, a percepção dos estudantes indica dificuldades no trabalho coletivo e com relação à autonomia, com alguns estudantes ainda declarando a necessidade de uma intervenção mais aguda do tutor.

Avaliando-se essas percepções dos estudantes, verifica-se de forma mais expressiva a contradição entre o que é proposto pelo RP I e II como ferramenta de criação de autonomia e a necessidade que eles indicam de maior diretividade, que transparece na proposta de "distribuição de tarefas" (c.f. Tabela 4). Notam-se também conflitos de percepção quanto ao processo de avaliação

\section{Estudante / Estudante}

\section{Difícil}

Complicada

Conflitante

Dificuldade de adaptação ao trabalho em grupo

Problemas de adaptação com a metodologia

Dificuldade no gerenciamento de tarefas e distribuição de responsabilidades

\section{Estudante / Tutor}

Relação horizontal

Relação participativa e próxima

Imposição de caminhos por parte do tutor

Afetividade

Percepção de um papel central do tutor

Sentimento de necessidade de direção das tarefas por parte do tutor

Tabela 2 - Sobre a Relação Estudante/Estudante e Estudante/Tutor. 


\section{Percepções Positivas}

Quebra do autoritarismo do professor

Construção coletiva do conhecimento

Iniciação aos métodos científicos

\section{Percepções Negativas}

Conflitos no relacionamento em grupo

Não percebem aspectos negativos

Falta de infraestrutura e de recursos para a pesquisa

Tabela 3 - Sobre as Mudanças na Forma de Conhecer/Aprender.

\author{
Rever processo de avaliação \\ Criar metodologia para divisão de tarefas gerenciada pelo tutor \\ Tutor mais diretivo \\ Maior estrutura física de sala e recursos para pesquisa (tecnológicos e de espaço) \\ Maior divulgação da pesquisa realizada \\ Preparação de tutores \\ Avaliação de tutores
}

Tabela 4 - Sobre Propostas de Melhorias no Processo de Aprendizagem.

coletiva do trabalho, sendo que algumas falas chegam a indicar essa responsabilidade de atribuição de notas ao tutor. A sugestão de preparação dos tutores e de avaliação mais consistente de sua atuação deve ser considerada como muito importante. Tais conflitos podem ser decorrentes do curto tempo de desenvolvimento de todo o processo (apenas quinze semanas), o que indicaria a necessidade de continuidade desses grupos em dois semestres seguidos com a mesma temática. Talvez isso auxiliasse no amadurecimento do grupo em relação à metodologia e também em relação à qualidade dos conteúdos desenvolvidos na pesquisa.

Finalmente, ao apresentar o processo desenvolvido no Ciclo Básico da EACH/USP, esperamos ter contribuído com uma reflexão sobre os nossos próprios propósitos na realização deste projeto pedagógico, bem como estimular outros colegas dispostos a incorporar estratégias de aprendizagem ativa em seus respectivos ambientes de aprendizagem.

\section{Notas}

1 Para conhecer na íntegra os três eixos norteadores das ações de aprendizagem adotadas pelo Ciclo Básico ver: http://each.uspnet.usp.br/cb/.

2 Para citar um exemplo, uma das turmas de RP II, com estudantes do curso de bacharelado em Lazer e Turismo, definiu como tema gerador "o lazer na metrópole". Contando com a mediação do tutor, os subgrupos iniciaram a busca por um problema de pesquisa que de alguma maneira se aproximasse de tal temática. O processo de definição dos problemas demorou bem mais do que o esperado, quatro semanas, e envolveu questões atuais, polêmicas e, dentre elas, prevaleceram as seguintes: a violência nas torcidas organizadas, o conteúdo agressivo das comédias stand-up, a relação entre lazer e hedonismo em uma importante rua de entretenimento de São Paulo (Rua Augusta), acessibilidade nos parques públicos e em museus da capital, hospitalidade em hotéis destinados ao público LGBT (Lésbicas, Gays, Bissexuais, Travestis, Transexuais e Transgêneros) e os impactos da Copa do Mundo de 2014 na cidade de São Paulo. Isso nos dá uma ideia da diversidade possível de temas a serem explorados, mesmo numa formação específica. 


\section{Referências Bibliográficas}

ANDRADE, Mariana Aparecida Bologna Soares de \& CAMPOS, Luciana Maria Lunardi. "Possibilidades e Limites da Prática da Aprendizagem Baseada em Problemas (PBL) no Ensino Médio". Revista Enseñanza de Las Ciencias, Barcelona, número extra, VII Congreso, 2005.

ARAÚJO, Ulisses Ferreira \& ARANTES, Valéria. "Comunidade, Conhecimento e Resolução de Problemas: o Projeto Acadêmico da USP Leste". In: ARAÚJO, U. F. \& SASTRE, G. Aprendizagem Baseada em Problemas no Ensino Superior. São Paulo, Summus, 2009, pp. 101-122.

FAUSTINO, Andréa Mathes. "Aplicação da Aprendizagem Baseada em Problemas na Graduação de Enfermagem: Revisão de Literatura". Revista Eletrônica Gestão \& Saúde, vol. 4, n. 1, 2013, pp. 1848-1859.

MIRANDA JUNIOR, Ubirajara José Picanço de. "Problem Based Learning como Metodologia Inovadora no Ensino de Graduação em Saúde". Revista Port. Med. Geral Fam., 2016, p. 32.

PARANHOS, Vania Daniela \& Mendes, Maria Manuela Rino. "Currículo por Competência e Metodologia Ativa: Percepção de Estudantes de
Enfermagem. Rev. Latino-Am. Enfermagem, vol. 18, n 1, jan./fev. 2010.

RESSEL, Lúcia Beatriz; BECK, Carmen Lúcia Colomé; GUALDA, Dulce Maria Rosa; HOFFMANN, Izabel Cristina; SILVA, Rosângela Marion da; SEHNEM, Graciela Dutra. "O Uso do Grupo Focal em Pesquisa Qualitativa". Texto Contexto Enferm., Florianópolis, vol. 17, n. 4, out./dez. 2008, pp. 779-86.

RIBEIRO, Luis Roberto de Camargo. Aprendizagem Baseada em Problemas (PBL): uma Experiência no Ensino Superior. São Carlos: EdUFSCar, 2008.

UNIVERSIDADE DE SÃO PAULO. Escola de Artes, Ciências e Humanidades. Ciclo Básico da $E A C H / U S P$, s.d. Disponível em: each.uspnet.usp.br/ cb. Acessado em 20 mar. 2017.

UVINHA, Ricardo Ricci. "Resolução de Problemas Aplicada ao Lazer e Turismo". In: LAJE, Beatriz Helena Gelas (org.). Lazer e Turismo: Conceitos e Reflexões. São Paulo: Plêiade, 2009, vol. 1, pp. 15-34.

VIGNOCHI, Carine; BENETTI, Carla da Silva; MACHADO, Carmem Lúcia Bezerra; MANFROI, Waldomiro Carlos. "Considerações sobre Aprendizagem Baseada em Problemas na Educação em Saúde", Rev. HCPA, vol. 29, n. 1, 2009, pp. 45-50.

Publicado em 31/03/2017. 